\title{
ONTOLOGY-ORIENTED INFERENCE-BASED LEARNING CONTENT MANAGEMENT SYSTEM
}

\author{
Mohamed Kholief, Nader Nada, Wedian Khedr \\ College of Computing and Information Technology, Arab Academy for Science, \\ Technology, and Maritime Transport, Alexandria, Egypt \\ kholieflgmail.com, dr.nader.nadalgmail.com, wedian.khedr@gmail.com
}

\begin{abstract}
The world is witnessing the electronic revolution in many fields of life such as health, education, government and commerce. E-learning is considered one of the hot topics in the e-revolution as it brings with it rapid change and greater opportunities to increase learning ability in colleges and schools. The fields of Learning Management Systems (LMS) and Learning Content Management Systems (LCMS) are full of open source and commercial products, however LCMS systems in general inherit the drawbacks of information system such as weakness in user expected information retrieval and semantic modelling and searching of contents \& courses. In this paper, we propose a new prototype of LCMS that uses the Semantic Web technologies and Ontology Reasoner with logical rules, as an inference engine to satisfy the constraints and criteria specified by a user, and retrieves relevant content from the domain ontology in an organized fashion. This enables construction of a user-specific course, by semantic querying for topics of interest. We present the development of an Ontology-oriented Inference-based Learning Content Management System OILCMS, its architecture, conception and strengths.
\end{abstract}

\section{KEYWORDS}

Learning Content, E-Learning Content Management, Ontology-driven Inference \& Semantic Web Services

\section{INTRODUCTION}

Ontology based semantic web technologies are promising solutions to give an explicit definition of the shared conceptualization of a certain domain and assembling E-Learning contents. In fact, ontologies constrain the set of possible mappings between symbols and their meanings. Obviously, the systematization of knowledge and its standardization provides an infrastructure for knowledge within a knowledge-based system. Ontologies integrated with learner profiles generate better results in an e-Learning LMS System and could be used in combination with multi-agent technologies for deriving personalized learning paths. Ontology can easily manage the knowledge domain of courses and allow more details of organizations and adaptation of the learning paths of students.

However LCMS inherits the drawbacks of information system such as poor user expected information retrieval and semantic search of contents \& courses. Also content reuse is often not possible and can not support building up extra information from existing information. For much of this time, learning software development has often been the result of individual ideas and initiative. LCMS systems suffer from a lack of interoperability and lack of standardization.

DOI : 10.5121/ijwest.2012.3309 
International Journal of Web \& Semantic Technology (IJWesT) Vol.3, No.3, July 2012

This paper proposes an ontology-based approach to integrate learning designs and learning objects. The motivation is to develop a new paradigm in LCMS capable of:

- Retrieving information with a high accuracy, and enhancing the delivering, sharing, and exchange.

- Enhancing existing information with deducted and implied information. Enrich the content in every process and iteration.

- $\quad$ Standardizing LCMS development cycle. The OILCMS (ontology- oriented Inferencebased LCMS) provides a platform where it is interoperable and integrated with other systems; the structured database, the generated rules and the concepts are standardized to be widely used.

We use Protégé and OWL Editor to construct domain ontology for its simplicity and popularity. While we use Jena APIs to interact with the Semantic Web Reasoning engine and Eclipse to implement our prototype.

The paper is organized as follows: Section 2 provides literature review about the concepts of LMS. Section 3 presents basic concepts about the Semantic Web and ontology theory and how to build ontology. Section 4 explains the conceptual framework developed OILCMS and detailed implementation of the system, and we explain a component-oriented approach to organize Learning content in domain ontology. Section5 provides related work and comparisons between them. Section 6 is conclusion and future work.

\section{LCMS - GENERAL CONCEPTS}

LMS is a software package, usually on a large scale, that enables the management and delivery of learning courses and resources to students. Most LMS systems are web-based to facilitate "anytime, anywhere" access to learning content and administration [6]. LMS are reporting systems and generally do not include ways to create new content or to deliver small packets of learning. LMS are created for tracking registration, attendance, class lists, grades, test results, class scheduling, and managing all learning events within an organization.

In contrast, the focus of an LCMS is on learning content. It gives authors, instructional designers, and subject matter experts the means to create e-learning content more efficiently. The primary business problem an LCMS tries to solve is to create just enough content just in time to meet the needs of individual learners or groups of learners. Rather than developing entire courses and adapting them to multiple audiences, instructional designers create reusable content chunks and make them available to course developers throughout the organization. This eliminates duplicate development efforts and allows for the rapid assembly of customized content. [2]

In earlier evaluations, authors used criteria and features that were not present in later evaluations like integration of CD material and on-line content, batch student and courses input, application sharing and search possibility. Some of these features were substituted by other things. Actually LCMS integration with other systems like registration information system, student information system, roster and other applications that contain data about students and courses replaced the need for manually inserting students and courses into an LCMS.

To build a LCMS, the content should also be associated to learners. Learning contents should be recognized and retrieved according to who the learner is and record information about the learner's experience. When the learners log on to the system and launch the content, they should 
International Journal of Web \& Semantic Technology (IJWesT) Vol.3, No.3, July 2012

be taken straight back to where they last left off. As the learners interact with the content, results are passed back to the system. The system can also change its behavior based on real time students' interactions. This would be based on test scores, learning style preferences, skills, communication abilities, organizational roles or any other relevant data. In particular the LCMS gets the right content to the right people and at the right time.

\section{Semantic Web and Ontology}

Semantic Web relies on structured sets of information and inference rules that allow it to "understand" the relationship between different data resources. Implementing the Semantic Web requires adding semantic metadata, or data that describes data, to information resources. This will allow machines to effectively process the data based on the semantic information that describes it. When there is enough semantic information associated with data, computers can make inferences about the data, i.e., understand what a data resource is and how it relates to other data.

Semantic Web is increasingly popular for various needs in educational institutions to assist management functions. In e-Learning systems, Semantic Web is also considered important to link and structure the learning resources. Such architecture will enable automated agents to reason about Web content, and perform intelligent inferences about that content to develop customized courses delivered just in time to the user, according to their preferences and needs. To achieve these goals we need to express the meaning (in terms of attributes) of the content by using Semantic Web technologies in several layers. [5] The following layers are the basic ones:

- The XML layer, which represents data;

- The RDF layer, which represents the meaning of data;

- The Ontology layer, which represents the formal common agreement about the meaning of data;

- The Logic layer, which enables intelligent reasoning with meaningful data.

The Semantic Web technologies help us to develop systems that gather E-Learning content from diverse sources; process, organize and share content with other humans or artificial agents using ontology. Such an approach makes contents machine-understandable and it becomes possible to develop automated Web services with those heterogeneous contents. Three important technologies for developing the Semantic Web are eXtensible Markup Language (XML), the Resource Description Framework (RDF) and Ontology Web Language (OWL), which classified data from multiple domain based on its properties and its relationship with other data. OWL is a stronger language as compared to RDF and has much greater machine interpretability. OWL is the relationships that concepts, objects and other entities hold with each other.

\section{Ontology theory}

The semantic web technology relies on ontology as its backbone. Ontology is a description (like a formal specification of a program) of the concepts and relationships that can exist for an agent or a community of agents. [1] The description can be in the form of classes (or sets), attributes (or properties), and relationships (or relations between members of the class). The ontologies are derived form the real world conceptualisation shared by humans as a knowldege base and implemented in digital described through machine readable languages such as OWL, XML.

The basic of ontology is often a taxonomy. Taxonomy is a way of classifying or categorizing a set of things using a hierarchical structure, which is a treelike structure, with the most general category as the root of the tree. Everything is derived from <owl:Thing > under which we have the classes, properties and instances. Classes are the each node which is an information entity that 
represents some object in the real world that is being modelled. The properties are the each link between two nodes in a taxonomy which represents a "subclassification-of" relation or a "superclassification-of" relationship. Individual instances of the classes are made which in together makes a knowledge-base. The results plotted are based on logical reasoning and linking. The use of ontologies is rapidly growing thanks to the significant functions they are carrying out in information systems and semantic Web and knowledge-based systems.

\section{Framework of OILCMS}

In this section, we explain the 3-Tier OILCMSs architecture after introducing the tools and techniques we used in developing our prototype. We also give a specific example of the system ontologies and the framework of the system. Our approach is equally effective for general purpose E-Learning content management as long as a domain-ontology is available to annotate contents.

\subsection{Tools and Techniques}

We used Protégé and OWL Editor [3] to construct domain ontology for its simplicity and popularity. We used Jena2 APIs and RDQL to interact with a generic Semantic Web Reasoner to implement our prototype. Jena is a Java framework for constructing Semantic Web applications and supports major ontology languages such as RDF/RDFS, DAML+OIL, and OWL (except OWLFull)[4]. We decided to use OWL as our ontology language because of its functionality, tool support (in particular Protégé 4.1 development tool) and status as an official W3C recommendation. SPARQL is the $\mathrm{W} 3 \mathrm{C}$ recommendation as a query language for $\mathrm{RDF}$ within the Jena framework. The purpose of SPARQL is to extract information from RDF graphs. This means that SPARQL only retrieves information stored in the model which contains a set of NTriples statements. SPARQL can process ontology in a number of languages including OWL. SPARQL, just like other RDF-based query languages (RDQL, SeRQL, SPARQL) are quite hard to give semantics with reference to OWL-DL and their expressivity is more powerful than OWLDL reasoners can cope with. A typical SPARQL query has the following form:

\section{SELECT ? $\mathrm{x}$ \\ WHERE (?x shortPrefix:localName "value") \\ USING shortPrefix FOR < URIprefix >}

?x is the variables to be returned to the application. In the WHERE clause, this specifies the graph pattern as a list of triple patterns. The USING clause defines an alias for the prefix of a URIs to simplify the URI. SPARQL can also query about predicates or objects too. The limitation of SPARQL is that there is no disjunction in the query. Though SPARQL is relatively simple in syntax, it is efficient for most of the ontology queries.

The reasoner can be used in combination with both Jena and OWL API libraries and also provides a DIG interface. Jena includes an RDFS reasoner (RDFSRuleReasoner) which supports almost all of the RDFS entailments described by the RDF Core working group. The reasoning provides functionalities validate and check consistency of ontologies, classify the taxonomy, check entailments and answer a subset of SPARQL queries. The reasoners can be described using RDF metadata which can be searched to locate reasoners with appropriate properties. 


\subsection{The 3-Tier Content Management Architecture}

In this section is described the architecture of the application. Today's most applications use 3tier client/server model like in Figure 1. The Repository tier stores both the content and its structure in an ontology. The Generic Ontology Reasoner engine is in the semantic tier between the Repository tier and End User Interface tier. The End User Interface tier interact with the content server through the Reasoner by specifying criteria (attribute-value pairs) and constraints; then the Reasoner locates the relevant contents (based on those conditions), and deliver the contents to the user/software agents in an organized fashion. This kind of system decomposition enables us develop large-scale software systems and reduce overall development time.

We use OWL to represent and link the content on the repository learning content server. The contents are needed to be annotated in advance. Annotation may also be done in a collaborative fashion. The domain ontology is to represent the domain knowledge. To illustrate the relationships or dependencies in this domain, we use 2 types of relationships, first is the direct relationship between content including its inverse relationship. Second is the indirect or transitive relationship such as prerequisite which means that it has inherited relationships.

The semantic tier interacts with users or agents and makes use of the Generic AI Reasoner to locate contents (based on user criteria) from the content server. We use Jena and SPARQL to query the content from the content server. The system loads the ontology model in OWL from the Content Server. Jena binds the model containing the original ontology. Then an RDF graph-base is ready for SPARQL query. The semantic tier generates queries based on user's or agent's request. Finally, the retrieved content presented to the user in an organized fashion.

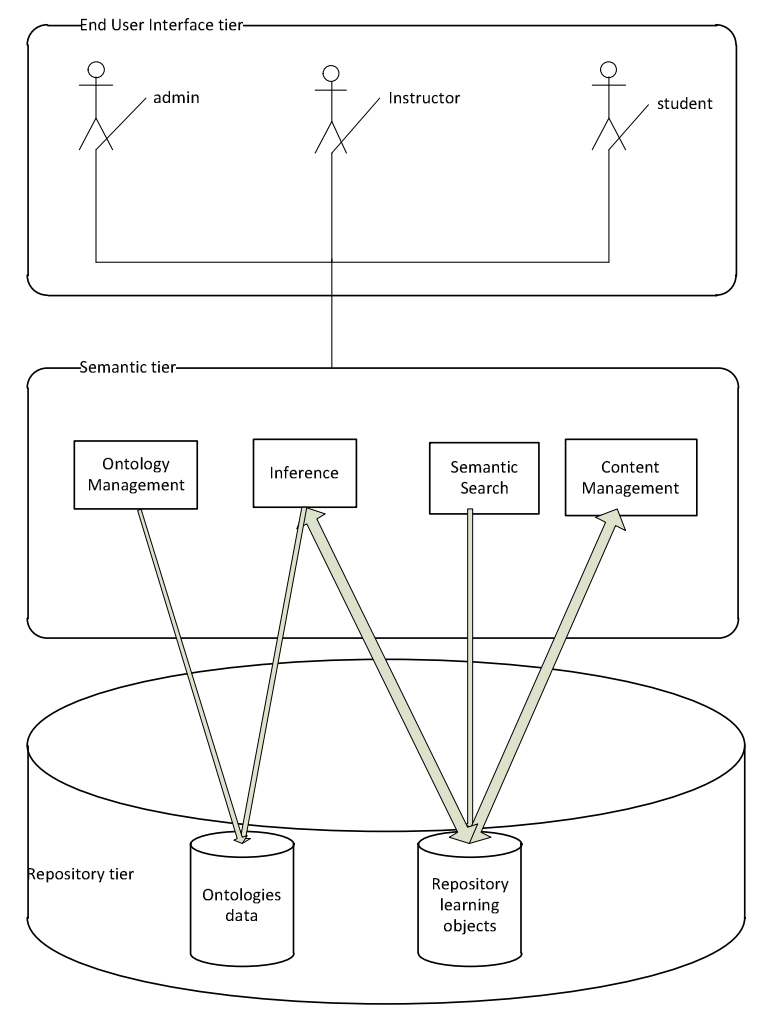

Figure 1. The OILCMS architecture. 


\subsection{Sequence diagram}

As shown in Figure 2, the sequence of steps carried out in the "query and retrieve" flow proceeds as follows: The user, after registration to the system, search for certain data. The Semantic-based LMS check if the query is valid; the system informs him if the query is invalid and/or if there was error in sending request. If it is valid, the system fetches the query at the repository data and user history repository, and check if data is existing; the system informs him if the data is not existing but if it exists, then the system retrieves the data and makes inference to the retrieving data by using the validation rules, saves the inference result's to the repository and adds the result to the original data retrieved and responses to the user.

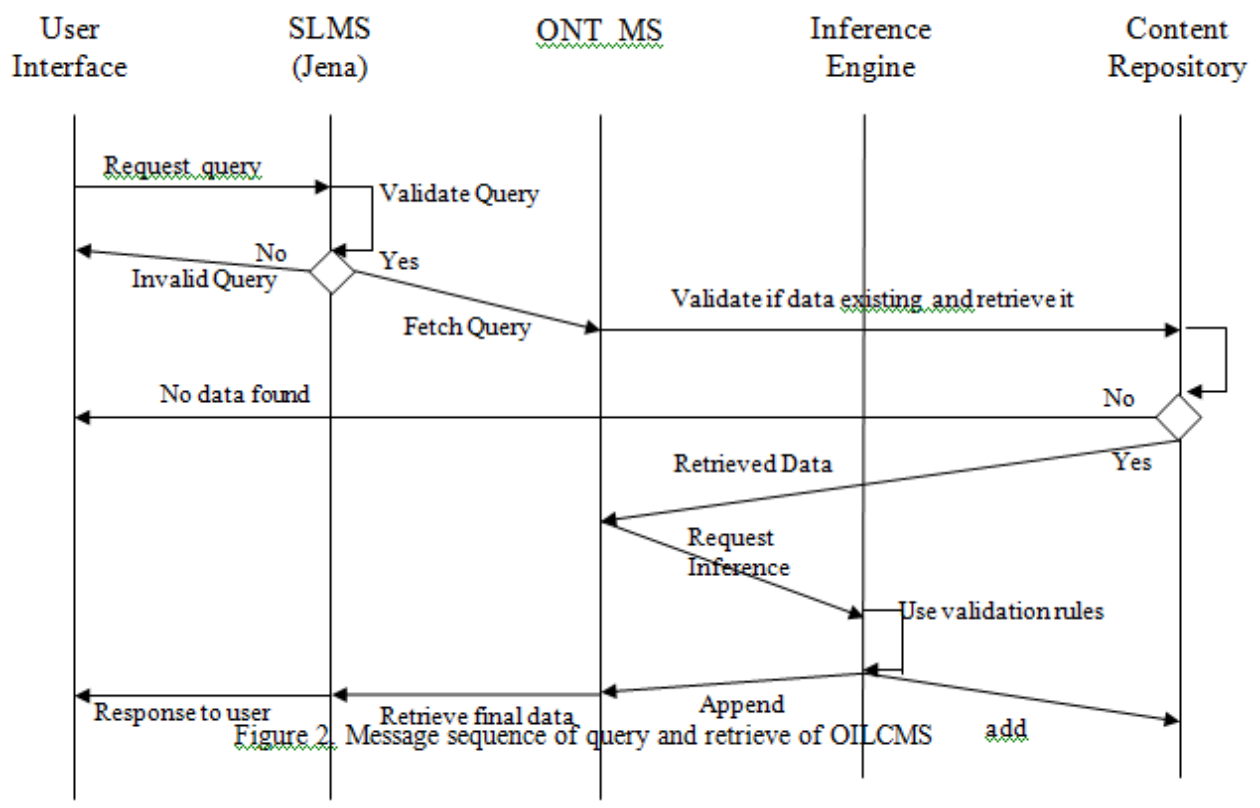

\subsection{Underlying Ontologies}

We based our system on the ontological framework to enable formal representation of learning content and its principle building blocks: different kinds of learning activities, participants in the learning process (learners, teachers, teaching assistants, and so on), and the learning content. The domains of the ontology defined can be reused by other ontologies, thus integrating several existing ontologies under one ontology describing a large domain.

The name of my ontology representing unique URI:

http://www.semanticweb.org/ontologies/2012/2/learning_resources.owl

\section{Defining Classes:}

We define three main classes namely Institutions, Learning Resources and Users. The Institutions class has been further subclassed into Colleges. The instances of these classes will be defined later. The Learning Resources class contains a list of all the courses as its subclasses and Users class contains a list of all kind of users (administrator, instructor, student ).

All the classes are subclassed under one class <owl:Thing $>$ which is the root of all the classes.

Defining properties: 
International Journal of Web \& Semantic Technology (IJWesT) Vol.3, No.3, July 2012

We define the necessary Object properties to link the classes and make it easier to have affected inference. And now the domain and the range of each property are defined to show which class they are pertaining to. We Show a sample of the Object Property as shown in Table1.

Also we define the necessary Datatype properties to identify the classes and give it some Characteristics.

Table 1. Sample of Object Properties

\begin{tabular}{|l|l|l|}
\hline Object Properties & Domain & Range \\
\hline HasCollege & Institutions & College \\
\hline HasDepartment & College & Department \\
\hline HasCourseComponent & Courses & $\begin{array}{l}\text { Course Content, Test, } \\
\text { Questionnaire }\end{array}$ \\
\hline HasAdminCourse & Courses & Course Administrator \\
\hline HasPre-request & Courses & Courses \\
\hline WorkAt & Instructor & Department \\
\hline CourseFieldis & Courses & Department \\
\hline
\end{tabular}

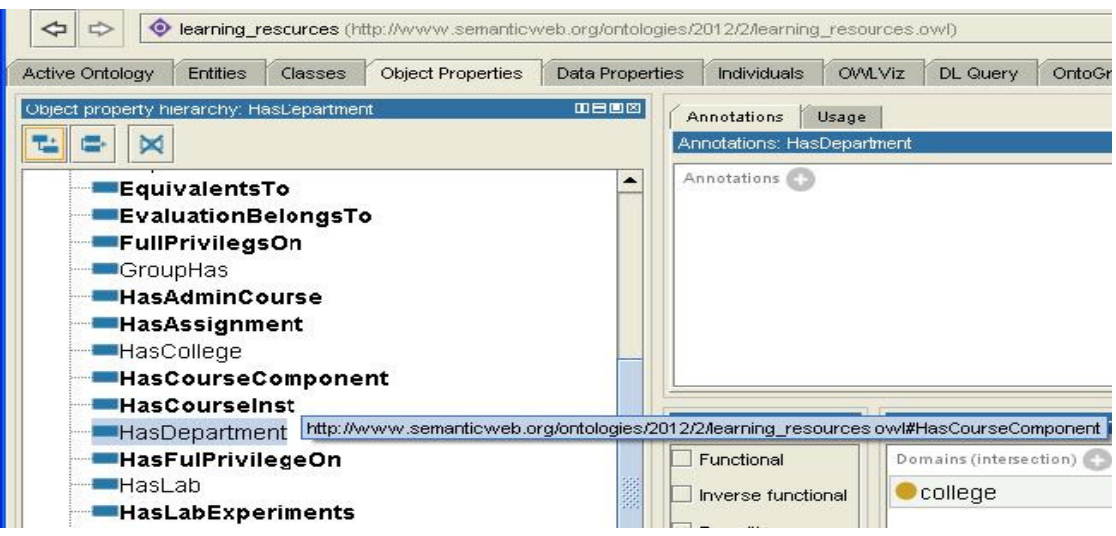

Figure 3. Object Properties

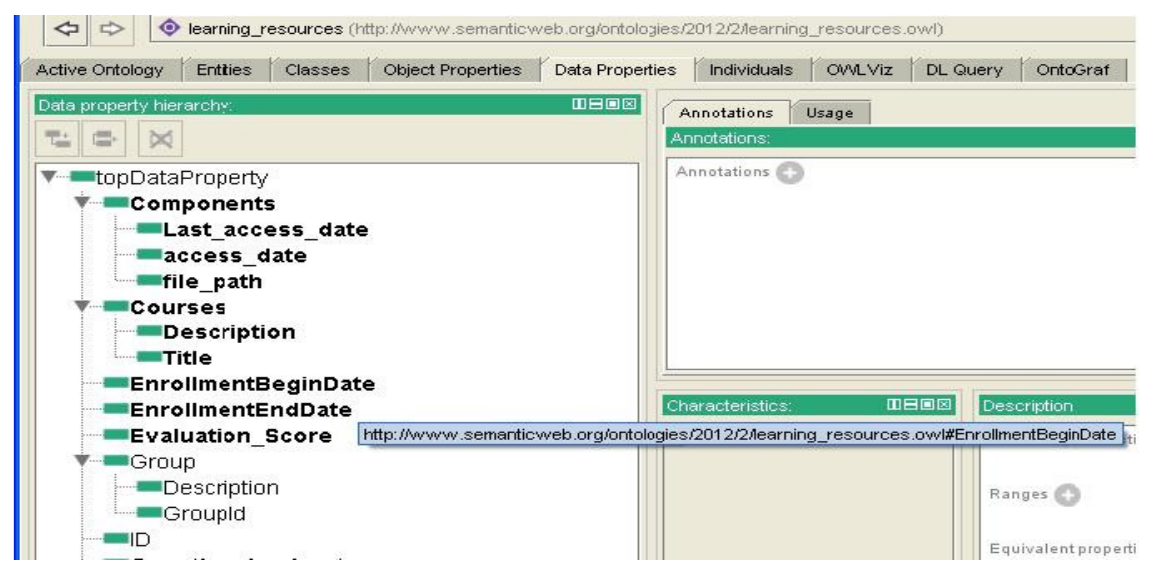

Figure 4. Datatype Properties

The following subclasses were made and examples of some instances generated from these subclasses: 
Table 2. Sample of Instances

\begin{tabular}{|l|l|l|l|}
\hline College & Department & Courses & Course Content \\
\hline Engineering & Computer_Engineering & DataBase & $\begin{array}{l}\text { Introduction to } \\
\text { Databases lecture }\end{array}$ \\
\hline Engineering & Computer_Engineering & DataBase & $\begin{array}{l}\text { Object Oriented } \\
\text { DBMSs lecture }\end{array}$ \\
\hline Engineering & Computer_Engineering & DataBase & $\begin{array}{l}\text { Entity Relationship } \\
\text { lecture }\end{array}$ \\
\hline
\end{tabular}

The learning resources ontology is the central part of this framework as shown on the graph using OntoGraf in Figure 5. It comprises several classes and properties aimed at formally representing learning content. We decide to separate the ontologies into two main parts: general course information and information about the learning content. The class courses are that one or more learners undertook while interacting with the system. And the other class is the course content. We've recognized a few types of this content: assignment, lab experience, notes, media, PowerPoint lecture, etc... In addition, we made subclasses for the courses. Class Course specification which includes information about the term, the field and the type of the course. Also the class course enrolment which includes information about the student's enrolment at certain course.

The ontology represents characteristics to all process of E-learning, such as some basic personal data and preferences, as well as learner-specific features, such as performance and different dimensions of learning styles.

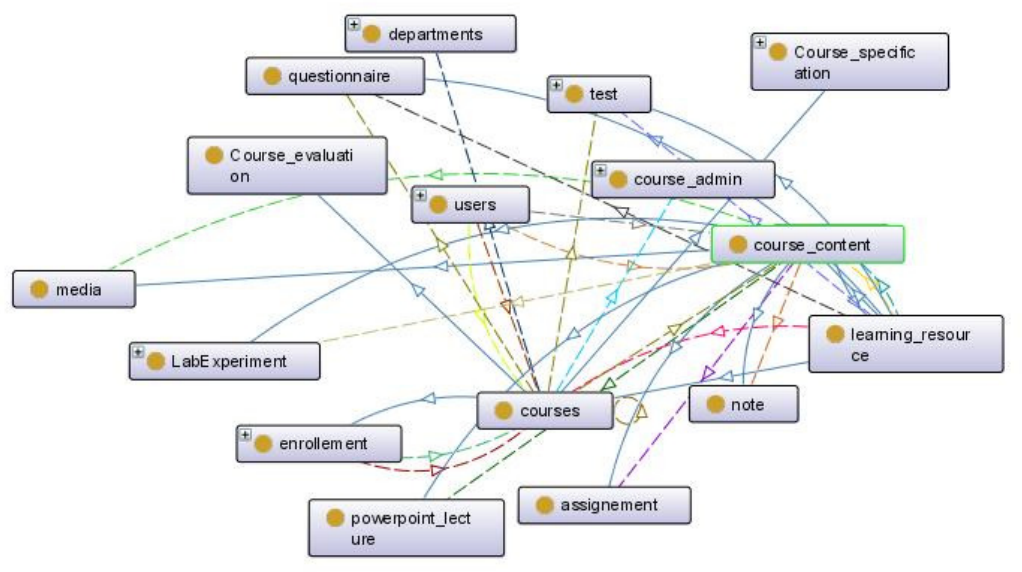

Figure 5. Proposed Ontology for the learning resource

\subsection{Class diagram}

A class diagram is UML structure diagram which describes the structure of the designed system by showing the system's classes, their features, constraints and relationships among the classes. The active entities of the proposed system are: Instructor, Courses, Course Content, Course administrator, and Student. The business domain entities of the system are Course Evaluation, Enrolment, Course Specification, and Test. The class diagram of our Proposed System built after a careful analysis of the requirements is shown in Figure 6. 
International Journal of Web \& Semantic Technology (IJWesT) Vol.3, No.3, July 2012

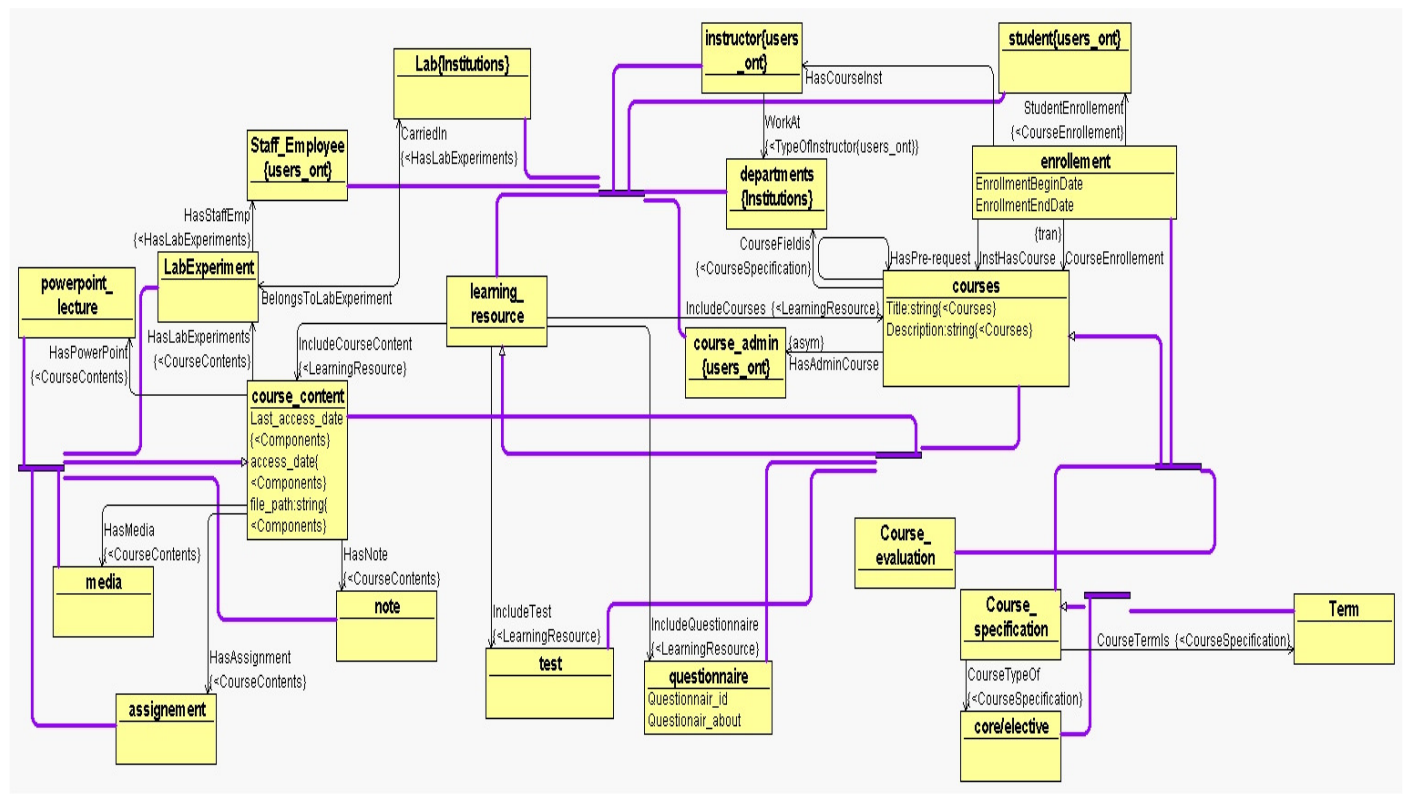

Figure 6. Learning Resources ontology - class diagram

\section{Logical Rules}

eprefix inst:

<http://www. semanticweb.org/ontologies/2012/2/Institutions.owl\#>.

aprefix user:

<http://www.semanticweb.org/ontologies/2012/2/users_ont.owl\#>

aprefix course:

<http: //www.semanticweb.org/ontologies/2012/2/learning_resources.owl\#>[r

ules1: (?x course:StudentEnrollment ?y) (?x course:CourseEnrollment ?z)

(?z course:HasCourseComponent ?t) ->(?y course:ReadPrivilegeOn ?t)]

[rules2:(?x course:HasPre-request ?y) (?y course:HasPre-request ?z) -> (?x

course:HasPre-request ?z)]

[rules3: (?x course:ContentBelongsToTopic ?y) (?y course:EquivalentsTo

?z) $->$ (?x course:ContentBelongsToTopic ?z)]

The above rules illustrate the semantic searching of courses content on the system. Rule rules 1 illustrates the privilege of the student to the course content which is enrollment. Rule rules2 illustrates all the pre-request courses of certain course. Rule rules 3 illustrates the topic of certain content, for example, the authentication lecture of course database is related to the topic security too.

\section{Related Work}

In this section, we will refer to E-Learning content management approaches and semantic web approach that are relevant to our work. Jovanovic et al. [7] They aimed to augment e-learning environments with semantic-rich awareness information. They intend educators to use this information when updating learning content for future courses. So they developed heuristics that enable both the intelligent analysis and conversion of LCMS log data into Semantic Web ontologies. Goran et al. [8] Intelligent LMSs (ILMSs) are bridge the gap between the modern approach to Web-based education based on LMSs and powerful but under used intelligent tutoring and adaptive hypermedia technologies. As the new Web generation, the Semantic Web 
has better conditions for composing and reusing learning materials. Ghoniemy et al. [9] This paper aims to design a simple, customized, learner-oriented e-learning system that facilitates the learning process. They aim to design a user-friendly, customized, learner-oriented e-learning system, and to create the material of a specific course conforming SCORM as a case study. Pramitasari et al. [10] This research focuses on development of student model ontology which is suited with the needs of learning process in Faculty of Computer Science University of Indonesia. The ontology covers the knowledge about student learning styles, student performance, and student data. Srimathi et al. [11] The research aims on ontology based approach of identifying such learning objects; which combines educational pedagogy and information technology through Instructional Design (ID). The primary aim of this study is to minimize the drop-out rate of elearning and promote the personalization. Colace et al. [12] This paper shows how to use Bayesian networks for easily mapping ontologies and presents a novel algorithm for building "lightweight" ontologies through them. Furthermore, the application of the proposed method in the assessment phase is illustrated in the form of a tool that builds the best assessment strategy according to the information inferred by the analysis of questionnaires. Cai et al. [13] The paper has demonstrated the natural use of Semantic Web technology to develop a ManuHub system for managing distributed manufacturing services with formal ontological support and friendly graphical user interfaces. Yıldırım et al. [14] This study proposes an automatic semantic content extraction framework. This is accomplished through the development of an ontology-based semantic content model and semantic content extraction algorithms.

In Table3, we present a comparison between related work and my system, we mean about Learning Technique that could be an e-learning web based or standard learning systems (LMSs, LCMSs).

Table 3. Comparison between related work

\begin{tabular}{|c|c|c|c|c|c|c|}
\hline Paper & $\begin{array}{l}\text { Semantic } \\
\text { Web }\end{array}$ & Language & $\begin{array}{l}\text { Learning } \\
\text { Technique }\end{array}$ & $\begin{array}{l}\text { Ontology } \\
\text { Based } \\
\text { Reasoning }\end{array}$ & $\begin{array}{l}\text { Rule Based } \\
\text { Reasoning }\end{array}$ & GUI \\
\hline $\begin{array}{l}\text { Jovanovic et } \\
\text { al. [7] }\end{array}$ & $\sqrt{ }$ & OWL & Standard & $\mathrm{X}$ & $\begin{array}{l}\text { Heuristics } \\
\text { Rules }\end{array}$ & $\sqrt{ }$ \\
\hline $\begin{array}{l}\text { Goran et al. } \\
{[8]}\end{array}$ & $\sqrt{ }$ & XML & Standard & $\mathrm{X}$ & $\mathrm{X}$ & $\mathrm{X}$ \\
\hline $\begin{array}{l}\text { Ghoniemy et } \\
\text { al. [9] }\end{array}$ & $\mathrm{X}$ & $\begin{array}{l}\text { Object } \\
\text { Oriented }\end{array}$ & $\begin{array}{l}\text { Web } \\
\text { Based }\end{array}$ & $\mathrm{X}$ & $\mathrm{X}$ & $\sqrt{ }$ \\
\hline $\begin{array}{l}\text { Pramitasari } \\
\text { et al. [10] }\end{array}$ & $\sqrt{ }$ & OWL & $\begin{array}{l}\text { Web } \\
\text { Based }\end{array}$ & $\sqrt{ }$ & $\begin{array}{l}\text { Logical } \\
\text { Rules }\end{array}$ & $X$ \\
\hline $\begin{array}{l}\text { Srimathi et } \\
\text { al. [11] }\end{array}$ & $\sqrt{ }$ & OWL & Standard & $\mathrm{X}$ & $\begin{array}{l}\text { Logical } \\
\text { Rules }\end{array}$ & $\mathrm{X}$ \\
\hline $\begin{array}{l}\text { Colace et al. } \\
{[12]}\end{array}$ & $\sqrt{ }$ & $\mathrm{X}$ & Standard & $\sqrt{ }$ & Bayesian & $\mathrm{X}$ \\
\hline $\begin{array}{l}\text { Cai et al. } \\
{[13]}\end{array}$ & $\sqrt{ }$ & OWL & Standard & $\sqrt{ }$ & $\mathrm{X}$ & $\sqrt{ }$ \\
\hline $\begin{array}{l}\text { Y1ldirım et } \\
\text { al. [14] }\end{array}$ & $\sqrt{ }$ & OWL & Standard & $\sqrt{ }$ & $\begin{array}{l}\text { Logical } \\
\text { Rules }\end{array}$ & $\mathrm{X}$ \\
\hline My work & $\sqrt{ }$ & OWL & Standard & $\sqrt{ }$ & $\begin{array}{l}\text { Logical } \\
\text { Rules }\end{array}$ & $\sqrt{ }$ \\
\hline
\end{tabular}




\section{Conclusions AND Future Work}

In this paper, we explained an ontology-oriented inference-based approach for E-Learning Content Management using Semantic Web technologies. The prototype can efficiently organize contents for a particular domain in ontology and, therefore, with the help of a generic Semantic Web Reasoner and created Jena rules, users can interact with the systems conveniently and can extract E-learning content efficiently. We used a specific domain (Database Course Contents) and a specific example (a 20-hour Application-oriented database course for students without a Foundation in databases) to demonstrate the essence of our approach in the context of personalized E-learning. We plan to apply our approach to web domain such as organizing the content available on a particular site such as the Moodle Course Management System (CMS). Integration of User Profiles with E-Learning Contents as proposed by Jovanovic' et al.[7] is also an interesting issue to explore under our 3-Tier Framework.

\section{REFERENCES}

[1] http://www.obitko.com/tutorials/ontologies-semantic-web/ontologies.html

[2] http://www.etraincenter.com/lms-lcms-compare.aspx

[3] Protégé, Protégé - Ontology Editor and Knowledge Acquisition System, retrieved March 2, 2006 from http://protege.stanford.edu/.

[4] Jena2, Jena - A Semantic Web Framework for Java, retrieved May 14, 2006 from http://jena.sourceforge.net/.

[5] Berners-Lee, "What is the Semantic Web," http://www.altova.com/semantic_web.html, cited on 12-10-08.

[6] What is the difference between a Learning Management System and a Learning Content Management System? 2010. www.guidetools.com/trainersguide/trainersguide8.htm.

[7] Jovanovic, J., Gasevic, D., Brooks, C., Devedzic, V., Hatala, M., Eap, T., \& Richards, G. (2007). Using Semantic Web Technologies to Analyze Learning Content. IEEE Internet Computing, 11(5), 45-53.

[8] Goran Šimić, Dragan Gašević, Vladan Devedžić, (2004). Semantic Web and Intelligent Learning Management Systems,

[9] Said Ghoniemy., Ashraf Fahmy., \& Sultan Aljahdali. A Dedicated Web-Based Learning System. Universal Journal of Computer Science and Engineering Technology 1 (2), 84 92, Nov.2010. (C) 2010 UniCSE, ISSN: 2219-2158.

[10] Leonny Pramitasari, A. Nizar Hidayanto, Siti Aminah, Adila A. Krisnadhi, and Meirna A. Ramadhanie. Development of Student Model Ontology for Personalization in an E-Learning System based on Semantic Web. In Proc. of the International Conference on Advanced Computer Science and Information Systems (ICACSIS) 2009, Universitas Indonesia, Depok, Indonesia.

[11] H. Srimathi, Srivatsa, S.K. Identification of ontology based learning object using instructional design.

[12] F.Colace, M.De Santo. Ontology for E-Learning: A Bayesian Approach. IEEE Transactions on Education, Volume 53 Issue 2, May 2010. 
International Journal of Web \& Semantic Technology (IJWesT) Vol.3, No.3, July 2012

[13] Cai, M., Zhang, W. Y. and Zhang, K., ManuHub: a Semantic Web system for ontology-based service management in distributed manufacturing environments. IEEE Transactions on Systems, Man, and Cybernetics Part A: Systems and Humans, in press, 2010.

[14] Y. Yıldırım, A. Yazıcı and T. Yılmaz. Automatic Semantic Content Extraction in Videos using a Fuzzy Ontology and Rule-based Model, IEEE Transaction on Knowledge and Data Engineering (TKDE) (2012).

[15] Lejla Abazi-Bexheti. Development of a learning content management system, WSEAS Transactions on Information Science and Applications, June 2008.

[16] http://jena.apache.org/documentation/inference/index.html 